absence of a detectable chromosome 15q11-13 anomaly does not change the AS phenotype. (Laan LAEM, Halley DJJ, den Boer ATh, et al. Angelman syndrome without detectable chromosome 15q11-13 anomaly: Clinical study of familial and isolated cases. Am I Med Genet March 1998;76:262-268). (Respond: LAEM Laan MD, Department of Neurology, Leiden University Medical Center, PO Box 9600, 2300 RC Leiden, The Netherlands).

COMMENT. The clinical manifestations of Angelman syndrome with a chromosome defect include delayed motor milestones, ataxic puppet-like gait, absent speech, paroxysmal laughter, mandibular prognathism, macrostomia, brachycephaly, hyperreflexia, truncal hypotonia, and scoliosis. Patients without detectable chromosomal anomaly have a higher frequency of truncal hypotonia and less frequent prognathism. Otherwise the AS phenotype is essentially the same. In a previous study at the University of Pisa, Italy, the puppet-like movements were a manifestation of cortical myoclonus. (Progress in Pediatric Neurology III, 1997;p 390).

\title{
RETT SYNDROME, SEIZURES AND NON-SEIZURES
}

Prolonged video/EEG/polygraphic monitoring was conducted in 82 Rett syndrome females, ages 2 to 30 years, at Baylor College of Medicine, Houston, TX. Clinical manifestations of reported seizures in $55(67 \%)$ patients included motor symptoms, generalized jerking, stiffening, behavioral changes, and staring. All had abnormal EEGs, epileptiform in 67 (81\%). During monitoring, electrographic seizures were recorded in only $13(16 \%)$ patients, and parents identified the clinical events confirmed as seizures in only 5 . Symptoms considered by parents as typical of 'seizures' were not associated with EEG discharges during monitoring in $23(42 \%)$ of the 55 patients considered initially to have seizures. These 'nonseizures' consisted of twitching, jerking, head turning, falling, trembling, staring, laughing, pupil dilatation, breath holding, and hyperventilation. The occurrence of epileptic seizures in RS is overestimated while true seizures may be under-recognized. (Glaze DG, Schultz RJ, Frost JD. Rett syndrome: characterization of seizures versus non-seizures. Electroencephalogr Clin Neurophysiol Jan 1998;106:79-83). (Respond: Dr Daniel G Glaze. Tel +1 713 7987388; fax +1 713 7984048).

COMMENT. Clinical seizures confirmed by electrographic video monitoring are frequently not recognized as seizures by parents of patients with Rett syndrome, whereas non-seizures are often considered to represent epileptic events. The authors have previously reported correlation of EEG abnormalities in Rett syndrome with the age-related changes in clinical stages. Serial records are characterized by a progressive deterioration, which further confuses the differentiation of seizures and non-seizures. Robb, Harden, and Boyd, at Great Ormond Street Hospital, London, fourd that EEG seizure discharges in 43 of 52 girls with Rett syndrome were not related to the onset of clinical seizures. These and other articles on Rett syndrome are reviewed in Progress in Pediatric Neurology I. 1991;pp513-528.

Growth failure in Rett syndrome was not explained by increased energy expenditure associated with repetitive involuntary movement, in a study of 14 girls at Baylor College of Medicine, Houston. (Motil KJ et al. L Pediatr Feb 1998;132:228-233).

Head circumference at birth in Rett syndrome patients was lower than that for a reference population in a study at the University of Western Australia, Perth. Birth weights were also lower, and $40 \%$ had reported perinatal difficulties and abnormalities in development in the first 6 months. Contrary to 
the criteria for diagnosis of Rett syndrome, head circumference at birth, perinatal period, and the first 6 months development may not be normal. (Leonard H, Bower C. Is the girl with Rett syndrome normal at birth? Dev Med Child Neurol Feb 1998;40:115-121).

\section{HEREDO-DEGENERATIVE DISEASES}

\section{CLINICAL, MRI, AND GENETIC FINDINGS IN BATTEN DISEASE}

The correlation of clinical, MRI and genetic factors in 36 patients with Batten disease (juvenile-onset neuronal ceroid lipofuscinosis) followed up for 25 years is reported from the Department of Paediatric Neurology, University of Helsinki, Finland, and other centers. Twenty seven patients were homozygous and 9 were heterozygous for the major mutation, a 1.02-kb deletion. All patients had vacuolated lymphocytes and positive rectal biopsy findings on the electron microscope. Visual failure occurred at a mean age of 5.8 years (range, 4-10 years) in all patients, and blindness developed between 6 and 20 years of age. Epilepsy began at 8 to 13 years in $92 \%$ of homozygous and $55 \%$ of heterozygous patients. Mental decline occurred slightly earlier (before 10 years in 50\%) in homozygous patients than in heterozygous (mean age 12). Neurological and MRI changes were milder in heterozygotes. Parkinsonian signs were noted in $30 \%$ of homozygous patients between 12 and 15 years, and in 22\% between 17 and 29 years. Extrapyramidal signs developed in only one heterozygous patient at 19 years. Speech failure correlated with onset of parkinsonism. Ataxia occurred in both homozygous and heterozygous patients before 15 years. Behavioral symptoms, aggression and depression, developed in 52\% of homoygotes and $33 \%$ of heterozygotes. Death of homozygotes occurred at a mean age of 24 years (range, 10-28 years). MRI abnormalities in homozygotes, cerebral atrophy and gray/white matter ratio changes, developed after 10 years of age in homozygotes. The 1.02-kb deletion in homozygous patients was always associated with mental and neurological handicaps, whereas the heterozygous phenotype was often benign and without intellectual deterioration. Whereas progression of visual impairment and epilepsy was highly concordant, progression of motor and mental deterioration was variable. Environmental and therapeutic factors, and modifying genes might influence the phenotype of Batten disease. (Jarvela I, Autti T, Lamminranta S, Aberg L, Raininko R, Santavuori P. Clinical and magnetic resonance imaging findings in Batten disease: Analysis of the major mutation (1.02-kb deletion). Ann Neurol Nov 1997;42:799-802). (Respond: Dr Jarvela, Laboratory of Human Molecular Genetics, Mannerheimintie 166, 00300 Helsinki, Finland).

COMMENT. Homozygous patients with Batten disease, having the major 1.02$\mathrm{kb}$ deletion, have mental and neurological handicap and a poor prognosis, whereas the heterozygous phenotype may be benign and intellectually normal. The diagnostic DNA test is of value in determining the severity and prognosis of the disease. MRI changes indicative of atrophy and progression of pathology may develop at and after 10 years of age.

\section{INFECTIOUS DISORDERS}

\section{MEASLES VACCINE AND ENCEPHALOPATHY}

The relationship between acute encephalopathy followed by permanent brain injury or death associated with further attenuated measles vaccine was evaluated in 48 children, ages 10 to 49 months, reported to the National Vaccine 